\title{
Diversidad de mamíferos en la cuenca media del río Tambopata, Puno, Perú
}

\author{
Mammal diversity in the middle basin of the river Tambopata, Puno, Peru
}

\begin{abstract}
1 Departamento de Mastozoología, Museo de Historia Natural, Universidad Nacional Mayor de San Marcos, Apartado 14-0434, Lima-14, Perú.

E-mail Víctor Pacheco: vpachecot@gmail.com

2 Facultad de Ciencias Biológicas, Universidad Nacional Mayor de San Marcos.
\end{abstract}

Presentado: 22/02/2011 Aceptado: $\quad 14 / 07 / 2011$ Publicado online: 25/08/2011

\section{Víctor Pacheco ${ }^{1,2}$, Gisella Márquez ${ }^{1}$, Edith Salas ${ }^{1}$ y Oscar Centty ${ }^{1}$}

\section{Resumen}

El presente trabajo documenta la diversidad de mamíferos en la cuenca media del río Tambopata, uno de los vacíos de información más importantes del Perú. Se evaluaron cinco localidades en Puno (San Fermín 850 m, Curva Alegre 950 m, Challohuma 1200 m, Yanahuaya 1600 m y Yanacocha 1985 m). El esfuerzo de captura fue de 7072 trampas noche y 201 redes noche. Se registraron 76 especies en el área de estudio de los cuales 16 especies estaban bajo alguna categoría de conservación. Los murciélagos y los roedores fueron los órdenes más diversos (67,5\%). Los índices de diversidad (H'y Simpson) de los mamíferos menores muestran una correlación negativa y moderada con la altitud. La Abundancia Relativa (AR) de los murciélagos tuvo una fuerte correlación negativa respecto de la altitud, mientras que los mamíferos pequeños terrestres una fuerte correlación positiva. Los marsupiales Marmosa (Micoureus) demerarae, Marmosops bishopi y Marmosops impavidus; el roedor Neacomys musseri; y los murciélagos Platyrrhinus albericoi, P. masu, P. nigellus, Eumops auripendulus y Cormura brevirostris son los primeros registros para el departamento de Puno. Las especies de roedores Oxymycterus juliacae y Akodon baliolus son consideradas validas. Las especies Sturnira lilium y Akodon baliolus fueron las que obtuvieron una mayor AR. Nuestros resultados, adicionados a literatura previa, indican que la cuenca del río Tambopata alberga actualmente 141 especies de mamíferos. Finalmente, recomendamos la extensión de la zona sur de la Zona de Amortiguamiento del PNBS hasta los bosques montanos de Yanacocha, para la protección de la fauna de la cuenca media del río Tambopata.

Palabras Clave: Diversidad, Mamíferos, Puno, Tambopata, Gradiente altitudinal, Conservación.

\section{Abstract}

This study documents the mammalian diversity in the Tambopata River middle basin, one of the most important information gaps in Peru. Five sites were evaluated in Puno (San Fermín 850 m, Curva Alegre 950 m, Challohuma $1200 \mathrm{~m}$, Yanahuaya $1600 \mathrm{~m}$, and Yanacocha $1985 \mathrm{~m}$ ). The trapping effort was 7072 trap-nights and 201 mistnet-nights. 76 species were recorded in the study area, including 16 species under some conservation's category. Bats and rodents were the most diverse orders $(67.5 \%)$. The diversity indices of Shannon-Wiener and Simpson for small mammals show a moderate and mild negative correlation with elevation, respectively. Meanwhile the altitude is negatively correlated with the relative abundance (RA) of bats, and positively with the RA of non-volant mammals. The marsupials Marmosa (Micoureus) demerarae, Marmosops bishopi and Marmosops impavidus; the rodent Neacomys musseri, and bats Platyrrhinus albericoi, P. masu, P. nigellus, Eumops auripendulus and Cormura brevirostris are first records for the department of Puno. Akodon baliolus and Oxymycterus juliacae are considered valid species. The species Sturnira lilium and Akodon baliolus were those with the highest RA. Our records, added to published and unpublished data, indicate that the Río Tambopata watershed is habitat of 141 species of mammals. Finally, we recommend the extension of the southern part of the buffer zone of the PNBS to include the montane forests of Yanacocha, to protect the unique middleelevation fauna of the Río Tambopata watershed.

Kewwords: Diversity, Mammals, Puno, Tambopata, Altitudinal gradient, Conservation.

\section{Introducción}

El Perú, con 508 especies, ocupa el quinto lugar en diversidad de mamíferos en el mundo (Pacheco et al. 2009) debido a la gran complejidad geográfica, climática y de ambientes; características relacionadas a la presencia de la Cordillera de los Andes y la Corriente Peruana. Esta diversidad varía según la región biogeográfica del país, con la mayor diversidad de especies en la Selva baja (Voss \& Emmons 1996, Emmons \& Feer 1997, Pacheco 2002, Pacheco et al. 2009), seguido por las Yungas y otras regiones biogeográficas (Pacheco et al. 2009).

Una de las zonas poco conocidas, en su diversidad de mamíferos es la cuenca media del río Tambopata, en el departamento de Puno. Esta zona se encuentra cerca al Parque Nacional Bahuaja Sonene (PNBS), área importante para el país por pertenecer al núcleo del Corredor de Conservación Vilcabamba-Amboró, uno de los corredores de conservación más importantes en el mundo (Ministerio de Agricultura 2003). El río Tambopata nace de los nevados del altiplano peruano- boliviano en La Paz y corre en dirección SO-NE hasta la localidad de San Fermín, cambiando de allí en dirección SE-NO hasta desembocar aguas abajo en el río Madre de Dios. La cuenca media, arriba de los $800 \mathrm{~m}$ de altitud incluye hábitats de bosque pre-montano tropical y bosque montano.

Esta zona se encuentra poco documentada, con pocos inventarios realizados por Hinojoza et al. (1987), Patton y Smith (1992) y Da Silva (1998), pero no se cuenta con un inventario completo de mamíferos. No obstante, Pacheco et al. (2007b), usando métodos deductivos e inductivo (Maxent) estimaron que dicha zona alberga una alta cantidad de especies endémicas.

En este contexto el presente trabajo contribuye a documentar la composición y diversidad de mamíferos de la cuenca media del río Tambopata durante dos expediciones realizadas, una en la época seca y otra en la época húmeda, y la revisión de bases de datos y literatura especializada, con los objetivos de: 1) Producir una lista de especies presentes para el área de estudio, 2) Determinar la abundancia relativa e índices de diversidad a lo largo de una gradiente altitudinal, 3) Evaluar el estado de conservación de las especies endémicas, raras, o listadas en alguna 


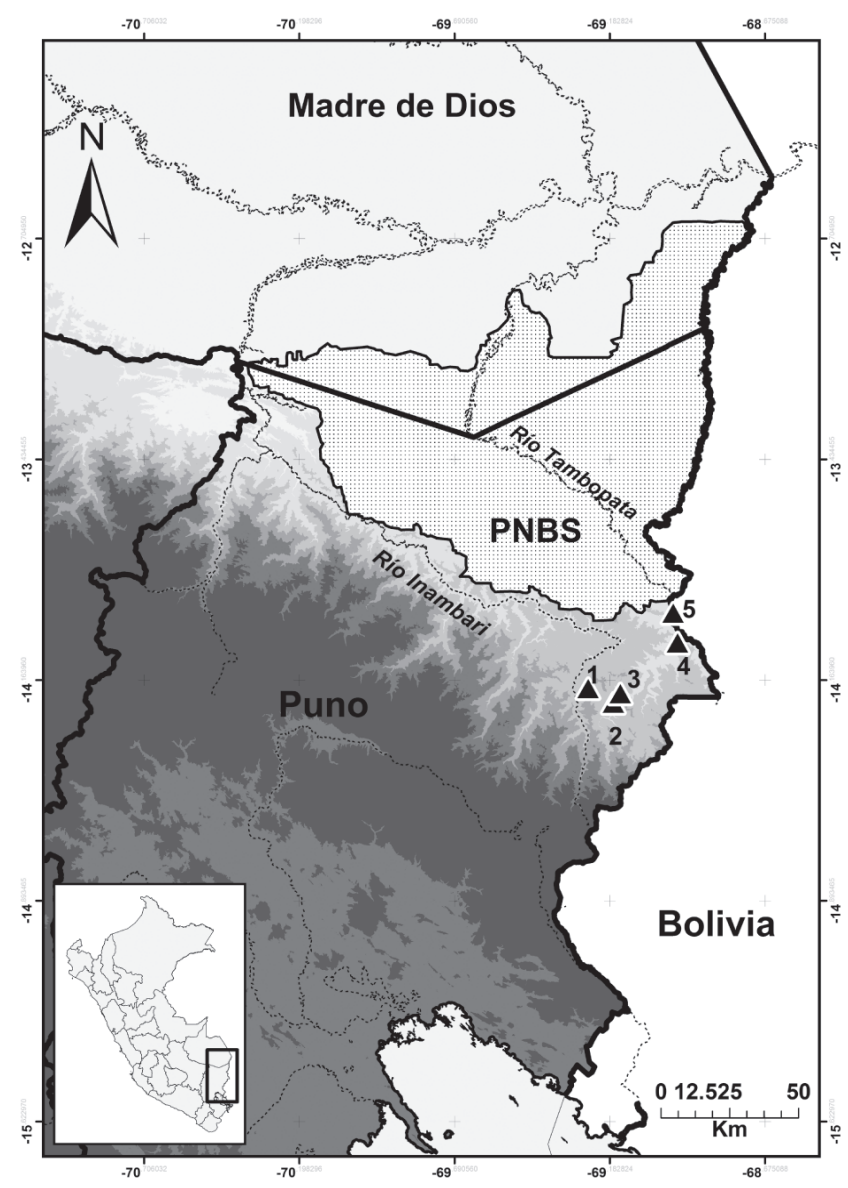

Figura 1. Ubicación de localidades muestreadas. PNBS= Parque Nacional Bahuaja Sonene. Localidades: 1=Yanacocha, $2=$ Yanahuaya, $3=$ Challohuma, $4=$ Curva Alegre y 5=San Fermín. categoría de conservación y 4) Sugerir la conservación de áreas basadas en criterios de diversidad, endemismo y presencia de especies amenazadas.

Área de estudio.- La cuenca del río Tambopata es una región muy extensa, cuya parte baja es mejor conocida por cruzar el PNBS. Para el presente trabajo se evaluaron cinco localidades en la cuenca media del río Tambopata, de $850 \mathrm{~m}$ a casi $2000 \mathrm{~m}$ de elevación, en el ańo 2009. En la primera etapa se evaluaron dos localidades con hábitats pre-montanos bajos: Curva Alegre y San Fermín, por aproximadamente 20 días, del 11 al 31 de mayo. En la segunda salida se evaluaron 3 localidades con hábitats pre-montanos y montanos: Yanacocha, Yanahuaya y Challohuma por aproximadamente 27 días, del 12 de agosto al 09 de septiembre (Fig. 1). Los datos de coordenadas y elevación para las 5 localidades se presentan en la Tabla 1.

\section{Localidades.-}

San Fermín (850 m).- En el distrito de San Pedro de Putina Punco, dentro de la Zona de Amortiguamiento (ZA) del PNBS. Incluye los hábitats de bosque pre-montano bajo, pacal y cafetal. El bosque pre-montano bajo muestreado cerca de la quebrada San Fermín presenta pendiente suave con pocos árboles grandes y rectos de $25 \mathrm{~m}$ de altura aprox., con abundantes lianas cubiertas de epífitas y musgos; y algunas palmeras; el sotobosque es relativamente abierto y el suelo presenta bastante humus. El pacal, compuesto por abundantes bambúes, está ubicado en el margen del río Tambopata, donde el suelo es arenoso pero por sitios es más pedregoso, en general presenta poca vegetación. Y el cafetal se encuentra representado por el monocultivo de café. El ambiente es en general muy húmedo.

Curva Alegre (950 m).- En el distrito de San Pedro de Putina Punco, dentro de la Zona de Amortiguamiento (ZA) del PNBS. Incluye los hábitats de bosque pre-montano bajo, ecotono bosque-río y cultivos de cítricos de naranja, mandarina,

Tabla 1. Ubicación de los lugares de muestreo en la cuenca media del río Tambopata.

\begin{tabular}{|c|c|c|c|c|c|}
\hline \multirow{2}{*}{ Localidad } & \multirow{2}{*}{ Unidad de vegetación } & \multirow{2}{*}{ Transectos } & \multirow{2}{*}{ Altitud (msnm) } & \multicolumn{2}{|c|}{ Coordenadas } \\
\hline & & & & Sur & Oeste \\
\hline \multirow{2}{*}{ Yanacocha } & \multirow{2}{*}{ Bosque montano } & $\begin{array}{l}\text { Pun-11 } \\
\text { Pun-12 }\end{array}$ & 1941 & $-14,1933$ & $-69,2558$ \\
\hline & & $\begin{array}{l}\text { Pun-13 } \\
\text { Pun-14 }\end{array}$ & 1985 & $-14,1925$ & $-69,2542$ \\
\hline \multirow{2}{*}{ Yanahuaya } & Pacal & $\begin{array}{l}\text { Pun-15 } \\
\text { Pun-16 }\end{array}$ & 1643 & $-14,2411$ & $-69,1711$ \\
\hline & Diversos cultivos (Bosque pre-montano) & $\begin{array}{l}\text { Pun-17 } \\
\text { Pun-18 }\end{array}$ & 1623 & $-14,2408$ & $-69,1711$ \\
\hline \multirow{2}{*}{ Challohuma } & Cultivos de cítricos & $\begin{array}{l}\text { Pun-19 } \\
\text { Pun-20 }\end{array}$ & 1200 & $-14,2097$ & $-69,1489$ \\
\hline & Bosque pre-montano & $\begin{array}{l}\text { Pun-21 } \\
\text { Pun-22 }\end{array}$ & 1265 & $-14,21$ & $-69,1453$ \\
\hline \multirow[t]{2}{*}{ Curva Alegre } & Bosque premontano bajo & $\begin{array}{l}\text { Pun-01 } \\
\text { Pun-02 } \\
\text { Pun-03 } \\
\text { Pun-04 }\end{array}$ & \multirow[t]{2}{*}{950} & \multirow[t]{2}{*}{$-14,0417$} & \multirow[t]{2}{*}{$-68,9614$} \\
\hline & Ecotono bosque-río & $\begin{array}{l}\text { Pun-05 } \\
\text { Pun-06 }\end{array}$ & & & \\
\hline San Fermín & 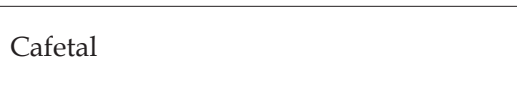 & $\begin{array}{l}\text { Pun-07 } \\
\text { Pun-08 } \\
\text { Pun-09 } \\
\text { Pun-10 }\end{array}$ & 850 & $-13,9436$ & $-68,9761$ \\
\hline
\end{tabular}


lima y toronja. El bosque pre-montano bajo muestreado presenta parches de bosque secundario pero se encuentra representado principalmente por cerros llenos de vegetación frondosa con presencia de árboles altos de unos $25 \mathrm{~m}$ de altura aprox. y palmeras, con sotobosque cerrado. La zona de ecotono se encuentra en las faldas de cerros abruptos al borde de un río no muy caudaloso, la vegetación es densa y herbácea con pocos arbolillos y el suelo es pedregoso con árboles caídos.

Challohuma (1200 m).- En el distrito de San Juan del Oro, fuera de la ZA del PNBS. Incluye los hábitats de bosque premontano y cultivos de cítricos de naranja, mandarina y limas dispersos por toda la zona. El bosque pre-montano presenta pendiente pronunciada, con algunos árboles grandes y rectos de hasta $15 \mathrm{~m}$ de altura y con un diámetro de grosor de $50 \mathrm{~cm}$ aproximadamente; con presencia de lianas cubiertas de epífitas y musgos. Suelo cubierto por abundantes herbáceas (algunas con tallo y hojas espinosas), con abundante humus y presencia de sitios pedregosos donde se forman algunos cuerpos de agua. Ambiente muy húmedo.

Yanahuaya $(\mathbf{1 6 0 0} \mathbf{~ m})$.- En el distrito de Yanahuaya, fuera de la ZA del PNBS. Incluye los hábitats de pacal y bosque premontano con presencia de diversos cultivos. Ambos hábitats son de pendiente suave. El pacal con abundantes bambúes y árboles delgados, la mayoría secos, con alturas de 6 a $12 \mathrm{~m}$ de altura aprox., se encuentra rodeando un cultivo de maíz; el suelo está generalmente cubierto con abundantes hojas secas de bambú y pasto, aunque por algunos sitios es pedregoso. El bosque premontano presenta un suelo pedregoso con presencia de árboles pequeños y delgados, donde se cultivan platanales, cafetales, piñas y yucas. En general el ambiente es seco.

Yanacocha $(\mathbf{1 9 8 5} \mathbf{m})$.- En el distrito de Alto Inambari, fuera de la ZA del PNBS. Incluye el hábitat de bosque montano. Cerros con presencia de parches de bosque secundario. La vegetación es semi frondosa, con árboles delgados, medianos y grandes de aprox. $15 \mathrm{~m}$ de altura, en su mayoría cubiertos de humus y lianas. Suelo limoso, muy húmedo, lleno de hojarasca y pastos, algunas zonas son rocosas, con presencia de cuerpos de agua sobre las rocas grandes y la pendiente va de moderada a fuerte. El área está cerca a una zona poblada pero hay pocos cultivos de cítricos. El ambiente en general es muy húmedo con densas neblinas por las mañanas.

\section{Material y métodos}

La metodología consistió en el uso simultáneo de trampas y redes en los diversos tipos de hábitats siguiendo la metodología detallada en Pacheco et al. (2007a). Se emplearon trampas de golpe (Museum Special y Víctor), trampas de caja (Sherman live trap), líneas pitfall y redes de niebla, todas ubicadas en sitios óptimos, dedicándose siete días de trampeo por localidad de muestreo. Las trampas fueron dispuestas en transectos de 30 estaciones, con un par de trampas por estación, por lo general una combinación de Víctor-Sherman o Museum SpecialSherman; y con una separación de aproximadamente $10 \mathrm{~m}$ entre las estaciones de trampeo, en un hábitat relativamente homogéneo. El cebo fue estándar, consistente en una mezcla de avena, mantequilla de maní, vainilla, miel de abeja, pasas y diferentes tipos de semillas para canarios. Las trampas fueron armadas en la tarde, revisadas en las primeras horas de la mañana y cebadas nuevamente en la tarde.
Tabla 2. Esfuerzo de muestreo de pequeños mamíferos no voladores y voladores por localidad evaluada en la cuenca media del río Tambopata. Donde: $\mathrm{BM}=$ bosque montano, $\mathrm{BPM}=$ bosque pre-montano, $\mathrm{BPB}=$ bosque pre-montano bajo, $\mathrm{E}=$ ecotono bosque-río, $\mathrm{CF}=$ cafetal, $\mathrm{PC}=$ pacal, $\mathrm{DC}=$ diversos cultivos, $\mathrm{CR}=$ cultivo de cítricos. $\mathrm{NT}=\mathrm{No}$ transectos. $\mathrm{EC}=$ esfuerzo de captura.

\begin{tabular}{llccccc}
\hline \multirow{2}{*}{ Localidad } & \multirow{2}{*}{$\begin{array}{c}\text { Unidad de } \\
\text { Vegetación }\end{array}$} & \multicolumn{2}{c}{ Trampas noche } & & \multicolumn{2}{c}{ Redes noche } \\
\cline { 3 - 4 } \cline { 6 - 7 } & & NT & EC & & NT & EC \\
\hline Yanacocha & BM & 4 & 1680 & & 2 & 51 \\
Yanahuaya & PC, DC & 4 & 1440 & & 2 & 42 \\
Challohuma & BPM, CR & 4 & 1432 & & 2 & 41 \\
Curva Alegre & BPB, E & 4 & 1440 & & 2 & 42 \\
San Fermín & CF, PC, BPB & 3 & 1080 & & 2 & 25 \\
TOTAL & & 19 & 7072 & & 10 & 201 \\
\hline
\end{tabular}

La evaluación de murciélagos se realizo utilizando redes de niebla de $12 \mathrm{~m}$ o $6 \mathrm{~m}$ de largo por $2,5 \mathrm{~m}$ de alto dispuestas en lugares óptimos, como caminos o quebradas, cerca de posibles refugios (e.g., cuevas, acantilados) y áreas de forrajeo (e.g., cerca de árboles con frutos, humedales, estanques). Las redes fueron revisadas cada hora aproximadamente, entre las 18:00 y las 24:00; cerrándose en la última revisión. Las redes fueron también cambiadas de lugar regularmente para cubrir los diferentes tipos de hábitats. En total se evaluaron 19 transectos para la captura de mamíferos no voladores y 10 transectos para voladores; con un esfuerzo de muestreo total de 7072 trampas noche y 201 redes noche (Tabla 2 ).

Los especímenes capturados se preservaron como pieles, cráneos y carcasas, o ejemplares completos en líquido (fijado en formol al $10 \%$ por 10 días y luego preservado en alcohol al $70 \%)$, y fueron identificados con un número de colector. Los especímenes se sacrificaron mediante dislocación cervical o por inyección intramuscular de una sobredosis del anestésico Ketamina al 10\%, en una dosis de $20 \mathrm{mg}$ por kilogramo de peso. Se registraron los datos del lugar de muestreo, tipo de hábitat, la estación y tipo de trampa. Adicionalmente, se tomaron las medidas estándar (longitud total, cola, pata y oreja; además longitud del antebrazo y el trago para los murciélagos), el peso, sexo, edad y la condición reproductiva de cada espécimen colectado. Los especímenes se encuentran depositados en el Departamento de Mastozoología del Museo de Historia Natural de la Universidad Nacional Mayor de San Marcos, Lima-Perú, para cualquier referencia o estudio posterior.

La presencia de los mamíferos de mayor tamaño fue evaluada mediante entrevistas y registros directos (observaciones, vocalizaciones, etc.) e indirectos (huellas, heces, madrigueras, etc.). Se entrevistó a pobladores o trabajadores locales, preferentemente varones residentes en el área de estudio, con ayuda de un modelo de preguntas preestablecidas e ilustraciones de especies probablemente presentes en la zona (ver Emmons \& Feer 1997 y Pacheco et al. 2007a). Las observaciones registradas en los lugares de muestreo se realizaron durante el establecimiento o la revisión de los transectos de mamíferos pequeños, o durante el desplazamiento entre localidades, anotándose para cada observación la hora, ubicación georeferenciada, tipo de vegetación y número de individuos. Las especies registradas a través de encuestas u observaciones no fueron incluidas en los análisis cuantitativos.

En el presente trabajo se sigue principalmente la nomenclatura taxonómica empleada en Pacheco et al. (2009) quienes 
incorporaron cambios sobre los presentados en Wilson y Reeder (2005) y Gardner (2008), sin embargo nosotros consideramos a los órdenes Pilosa y Cingulata como subórdenes de Xenarthra siguiendo la taxonomía de Van Dijk et al. (1999), Springer et al. (2003), Nishijara et al. (2006) y Nishijara et al. (2009). Los nombres comunes empleados siguen a Pacheco et al. (2009). Para un estimado completo de la cuenca del Río Tambopata se incorporan los registros de Ascorra y Orihuela (in litt.) y Foster et al. (1994), cuya nomenclatura fue previamente actualizada.

Para estandarizar el esfuerzo de muestreo, este se expresa como trampas noche para el caso de los pequeños mamíferos no voladores y como redes noche para los mamíferos voladores. Se determina la abundancia relativa (AR) de una especie en relación al esfuerzo de captura empleado. Así, la abundancia relativa de los marsupiales y roedores es el número de individuos capturados (incluyendo los liberados) por cada 100 trampas noche, y el de murciélagos es el número de individuos capturados (incluyendo los liberados y excluyendo los recapturados) por cada 10 redes noche. El esfuerzo de captura por cada localidad se presenta en la Tabla 2.

La diversidad de los lugares de muestreo fue estimada en base a la riqueza de especies $(S)$, los índices de diversidad de Shannon-Wiener, de Simpson y equitatividad (Krebs 1989). Se utilizó el coeficiente de correlación de Pearson para evaluar la relación entre la abundancia relativa y la diversidad de mamíferos con la elevación.

El estado de conservación de las especies, especialmente las endémicas y amenazadas, fueron evaluadas con el contexto de la legislación vigente (Decreto Supremo No 034-2004-AG), y los acuerdos internacionales (CITES 2010, IUCN 2008).

\section{Resultados}

\section{Composición de especies}

Se obtuvieron 471 especímenes, de los cuales 143 especímenes fueron liberados después de haber determinado la especie y tomado sus medidas. Se obtuvieron también 17 observaciones de mamíferos medianos o grandes, además de varias entrevistas consideradas confiables.

Las revisiones taxonómicas para la determinación de las especies confirman la presencia de 76 especies de mamíferos nativos para las cinco localidades evaluadas en la cuenca media del río Tambopata, las que incluyen cinco didelfimorfios, tres xenarthros, ocho primates, 18 roedores, un lagomorfo, 33 murciélagos, cuatro carnívoros, un perisodáctilo y tres cetartiodáctilos (Apéndice 1).

Como era de esperarse en el área de estudio, los murciélagos (33 especies, 42,8\%) y los roedores (19 especies, 24,7\%) fueron los órdenes más diversos, sumando ambos el 67,5\% del total de especies, mientras que los otros siete órdenes reportados acumulan solo el 32,5\% de especies. Dentro de estos órdenes, las familias Phyllostomidae y Cricetidae fueron las más representadas ( 87,50 y 52,63\% respectivamente). Los lagomorfos y perisodáctilos, cada uno con una sola especie, fueron los órdenes menos diversos. Por otro lado, a nivel de familias, los roedores, los murciélagos y los carnívoros presentaron la mayor diversidad, con siete, tres y tres familias respectivamente.

Se sigue aquí a Allen (1900) al reconocer a Oxymycterus juliacae como especie valida. Un ejemplar capturado en Challohuma presenta la nariz ancha, en forma de trompa y con la punta levantada hacia arriba, el rostrum proporcionalmente ancho y otras características que concuerdan con Allen (1900) y que no están presentes en ejemplares de $O$. inca encontrados en la misma cuenca a menor elevación. Por otro lado, Akodon baliolus fue descrito como A. aerosus baliolus por Osgood (1915) en base a una serie de Inca Mines, río Inambari, Puno. Sin embargo, Smith y Patton (2007) demostraron que aerosus no es monofilético. Previamente, Patton y Smith (1992) conservativamente incluyeron baliolus, así como surdus, dentro de aerosus, remarcando que uno o ambos podrían ser especies válidas distintas de aerosus. Patton et al. (1990) también documentaron variaciones extensivas en número diploide entre poblaciones de aerosus, con 2n 40 en el departamento de Ayacucho, 22 en el departamento de Cuzco y 38 en Puno. Además, se sustenta aquí el reconocimiento de baliolus al encontrar que este taxa tiene los nasales cortos, que no se extienden mas allá de los premaxilares, lo cual no es visto en muestras de aerosus s.s. de Ecuador y otras poblaciones del norte y centro del Perú. Estos argumentos taxonómicos son tratados en extenso en una publicación aparte.

Entre las especies registradas, varios son nuevos registros regionales o proporcionan información adicional sobre la especie. Los marsupiales Marmosa (Micoureus) demerarae y Marmosops bishopi, capturados en San Fermín, Yanahuaya, Yanacocha; y Challohuma respectivamente, son los primeros registros para el departamento de Puno. El marsupial Marmosops impavidus, capturado en Yanahuaya y Challohuma, representa el primer registro para el departamento de Puno y el más austral en su distribución. El marsupial Monodelphis peruviana, es una especie muy rara siendo nuestro ejemplar colectado en Curva Alegre el segundo registro conocido para Puno. El roedor Neacomys musseri capturado en San Fermín, viene a ser el primer registro para Puno y el más austral conocido para la especie. Los murciélagos Eumops auripendulus capturado en Challohuma, Platyrrhinus nigellus capturado en Curva Alegre y el murciélago Platyrrhinus masu capturado en San Fermín, Curva Alegre, Yanahuaya y Yanacocha, representan también primeros registros de sus especies para el departamento de Puno. El murciélago Platyrrhinus albericoi, capturado en San Fermín y Curva Alegre, viene a ser el primer registro para el departamento de Puno a menor elevación. Se registra Carollia manu a 850 m en San Fermín, $630 \mathrm{~m}$ más abajo que el rango de elevación mínimo conocido (1480 m, Pacheco et al. 2004). Esta especie fue encontrada en simpatría con C. perspicillata, C. brevicauda y C. benkeithi. El murciélago Histiotus velatus capturado en Challohuma es una especie rara y nuestros registros son los primeros de su especie para Puno. Adicionalmente también encontramos en la colección MUSM el primer registro de Cormura brevirostris para el departamento de Puno.

Al compararse las localidades evaluadas (Apéndice 1, Fig. 2), se observa que San Fermín y Curva Alegre, ambas a menor elevación y en bosque pre-montano bajo tropical, registraron la mayor diversidad de especies con 40 y 38 especies respectivamente, mientras que Yanahuaya fue el lugar menos diverso con 21 especies. A nivel de familias y órdenes, Curva Alegre es la localidad más diversa (Apendice 1). Yanahuaya, con un hábitat de pacal en un bosque pre-montano seco, resalta por ser la localidad más diversa dentro del orden Rodentia. San Fermín y Curva Alegre también presentaron una notable diversidad dentro del orden Chiroptera (Fig. 2). También se observa que la mayor 


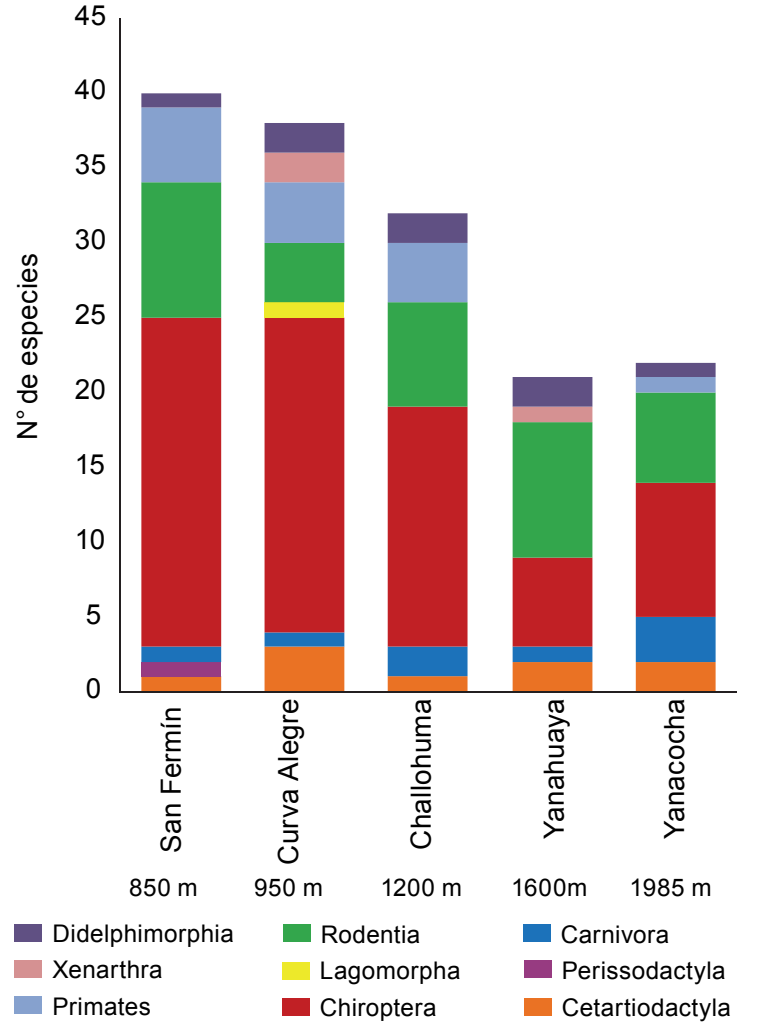

Figura 2. Número de especies de mamíferos registradas por localidad en la cuenca media del río Tambopata. Se considera el total de registros por captura, encuesta, avistamiento, huellas, heces y otros.

diversidad del orden Primates se concentra a menores elevaciones en las localidades de San Fermín, Curva Alegre y Challohuma a 850, 950 y 1200 m respectivamente. En general, el número de especies de mamíferos en la cuenca media del río Tambopata disminuye mostrando una correlación negativa significativa $(\mathrm{P}$ $<0,05)$ con el aumento de la elevación $(r=-0,93)$.

El oso de anteojos (Tremarctos ornatus) registrado por heces y marcas de sus garras en un árbol en Yanacocha y Challohuma respectivamente son dos reportes indirectos de la presencia del oso andino en la cuenca media del río Tambopata, confirmando su actual distribución en el departamento de Puno. Además el registro de Challohuma evidencia ser hasta el momento, el registro más austral confirmado para la distribución del oso andino en el Perú (Márquez \& Pacheco 2010).

Finalmente, se presenta una lista actualizada de la mastofauna presente en el área del Tambopata basada en nuestros registros y de publicaciones previas (Apéndice 1), sumando un total de 141 especies de mamíferos; 114 presentes en la cuenca baja y 76 en la cuenca media; mostrando que la mayor diversidad de mamíferos se halla a elevaciones bajas.

\section{Abundancia relativa}

Se obtuvieron datos de abundancia relativa (AR) para las especies capturadas y se analizaron para el grupo de mamíferos no voladores (marsupiales y roedores) y voladores (murciélagos). En el primer grupo se observa que la localidad de Yanahuaya registra la mayor AR seguida de Challohuma y Yanacocha con valores similares. San Fermín registra los valores de AR más bajos. Al parecer AR más altos están relacionadas con hábitats de elevaciones altas dado que muestran una correlación positiva moderada con la elevación $(r=0,72)$. En los bosques pre-
Tabla 3. Abundancia relativa de mamíferos no voladores por localidad evaluada en la cuenca media del río Tambopata.

\begin{tabular}{|c|c|c|c|c|c|}
\hline 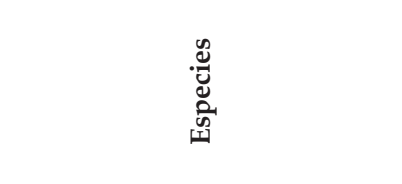 & 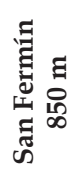 & 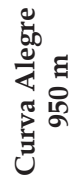 & 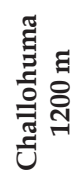 & 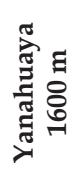 & 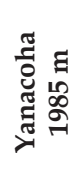 \\
\hline Akodon baliolus & & & 0,91 & 2,08 & 0,95 \\
\hline Euryoryzomys nitidus & 0,28 & 0,83 & & & \\
\hline Hylaeamys perenensis & & & & 0,07 & \\
\hline Lenoxus apicalis & & & & 0,07 & 0,24 \\
\hline Neacomys musseri & 0,09 & & & & \\
\hline Neacomys spinosus & & & 0,07 & & 0,24 \\
\hline Nephelomys keaysi & & & & 0,14 & \\
\hline Oecomys bicolor & 0,09 & & 0,07 & & \\
\hline Oligoryzomys destructor & & & 0,35 & 0,07 & 0,10 \\
\hline Oxymycterus juliacae & & & 0,07 & & \\
\hline Marmosa (Micoureus) demerarae & 0,09 & & & 0,07 & 0,18 \\
\hline Marmosops bishopi & & & 0,35 & & \\
\hline Marmosops impavidus & & & 0,07 & 0,07 & \\
\hline Marmosops noctivagus & & 0,07 & & & \\
\hline Monodelphis peruviana & & 0,07 & & & \\
\hline Total & 0,56 & 0,97 & 1,89 & 2,57 & 1,79 \\
\hline
\end{tabular}

montanos de Yanahuaya el roedor Akodon baliolus alcanzó la más alta AR, mientras que a menores elevaciones en San Fermín y Curva Alegre Euryoryzomys nitidus fue el más abundante. Los didelfimorfios Marmosops noctivagus y Monodelphis peruviana fueron más bien raros, con AR muy baja (Tabla 3).

Para los murciélagos, la localidad de Curva Alegre presenta la mayor abundancia seguida de San Fermín. Yanahuaya registra los valores de AR más bajos. La correlación negativa moderada entre la AR y la elevación $(r=-0,76)$ sugiere que la $A R$ es más alta a menores elevaciones (Tabla 4). En el bosque pre-montano bajo tropical de San Fermín las especies Sturnira lilium y Carollia benkeithi fueron las más abundantes, mientras que en Curva Alegre y Yanacocha, Sturnira lilium es la especie con mayor AR. Esta última especie es también la que registra la mayor AR en toda el área de estudio. En Challohuma, Uroderma bilobatum e Histiotus velatus son las especies más abundantes, mientras que para Yanahuaya es Carollia brevicauda.

Los registros realizados por avistamientos, encuestas, huellas, vocalizaciones, heces, etc. proporcionaron un estimado cualitativo de abundancia. En San Fermín se han realizado avistamientos de pichicos Saguinus fuscicollis, machines Cebus apella, frailecillos Saimiri boliviensis y maquisapas Ateles chamek; los cuales son considerados frecuentes por los pobladores locales. También se registraron vocalizaciones casi diarias del mono coto Alouatta sp., probablemente $A$. sara. en Pampa Grande, poblado cercano a San Fermín y en Challohuma se observó una mascota del machín negro Cebus apella, de igual forma para esta última localidad se observó una mascota del pichico Saguinus fuscicollis y una mascota de una cría del tocón de Madidi Callicebus aureipalatii, el cual provino de zonas más bajas del río Tambopata, de la selvas de San Pedro de Putina Punco, según comentaron sus dueños. En San Fermín se registraron todos los avistamientos de ardillas reportadas en el presente trabajo. En todas las localidades excepto en Yanacocha se registraron vocalizaciones diarias del cono-cono boliviano Dactylomys boliviensis, considerado por nosotros como una especie localmente abundante. 
Tabla 4. Abundancia relativa de mamíferos voladores por localidad evaluada en la cuenca media del río Tambopata.

\begin{tabular}{|c|c|c|c|c|c|}
\hline Especies & 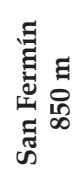 & 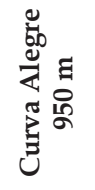 & 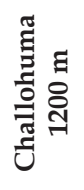 & 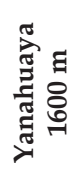 & 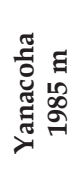 \\
\hline Desmodus rotundus & 0,40 & 0,71 & & & \\
\hline Anoura caudifer & 0,40 & 0,48 & & & \\
\hline Anoura geoffroyi & & & 0,98 & & 1,18 \\
\hline Glossophaga soricina & 0,8 & 0,48 & 0,49 & & \\
\hline Chrotopterus auritus & & 0,24 & & & \\
\hline Phyllostomus hastatus & & 0,24 & & & \\
\hline Carollia benkeithi & 4,4 & 0,95 & & & \\
\hline Carollia brevicauda & 2,8 & 0,95 & 0,73 & 0,95 & 1,57 \\
\hline Carollia manu & 0,40 & & & & \\
\hline Carollia perspicillata & 4 & 2,62 & 0,24 & 0,24 & \\
\hline Artibeus glaucus & 0,40 & 1,67 & 0,24 & 0,24 & 1,37 \\
\hline Artibeus lituratus & 0,40 & & & & \\
\hline Artibeus obscurus & 2,40 & 0,24 & & & \\
\hline Artibeus planirostris & 2,40 & 1,19 & 0,24 & 0,24 & 0,78 \\
\hline Chiroderma salvini & & & 0,24 & & \\
\hline Chiroderma trinitatum & 0,40 & 0,24 & & & \\
\hline Mesophylla macconnelli & 0,40 & & & & \\
\hline Platyrrhinus albericoi & 0,80 & 0,24 & & & \\
\hline Platyrrhinus brachycephalus & 0,80 & 0,71 & & & \\
\hline Platyrrhinus incarum & 2,40 & 0,24 & & & \\
\hline Platyrrhinus infuscus & 0,80 & & & & \\
\hline Platyrrhinus masu & 2 & 0,95 & & 0,24 & 0,98 \\
\hline Platyrrhinus nigellus & 1,20 & 1,19 & & & \\
\hline Sturnira erythromos & & 1,67 & & & 0,20 \\
\hline Sturnira lilium & 4,40 & 19,76 & 0,24 & 0,48 & 3,14 \\
\hline Sturnira magna & & 0,71 & & & \\
\hline Sturnira oporaphilum & 3,60 & 5,24 & 0,24 & & 0,20 \\
\hline Uroderma bilobatum & 0,40 & 0,95 & 1,46 & & \\
\hline Eumops auripendulus & & & 0,49 & & \\
\hline Tadarida brasiliensis & & & 0,24 & & 0,20 \\
\hline Histiotus velatus & & & 1,46 & & \\
\hline Myotis albescens & & & 0,24 & & \\
\hline Myotis nigricans & 0,40 & & & & \\
\hline Total & 36,40 & 116,67 & 34,44 & 2,38 & 9,61 \\
\hline
\end{tabular}

\section{Índices de diversidad}

Los valores de los índices de diversidad de Shannon-Wiener $\left(\mathrm{H}^{\prime}\right)$ y de Simpson $(1-\mathrm{D})$, se obtuvieron de los individuos capturados de cada localidad y se presentan en la Tabla 5. Se observa que San Fermín (850 m) y Challohuma (1200 m) son las localidades con mayor diversidad $\left(\mathrm{H}^{\prime}=4,20\right.$ y 3,52 respectivamente), mientras que Yanahuaya (1600 m) y Curva Alegre $(950 \mathrm{~m})$ son los de menor diversidad $\left(\mathrm{H}^{\prime}=2,17\right.$ y 3,22 respectivamente), reflejando el peso que da el índice a la riqueza de especies. En cambio con el índice de dominancia de Simpson a excepción de Yanahuaya que registra el valor más bajo $(1-\mathrm{D}$ $=0,58)$, las otras localidades presentan valores bastante altos y similares a pesar de las grandes diferencias en el número de especies. Se observa que los ambientes pre-montanos y montanos de la cuenca media del río Tambopata muestran una correlación negativa débil entre los valores de H’y la elevación $(r=-0,50)$, mientras que con el índice de dominancia la correlación es aún más débil $(r=-0,27)$ por ello no se concluye que exista una asociación lineal entre la diversidad y la elevación. En cuanto a la equidad (J') se observa que fue alta en todos los lugares con excepción de Curva Alegre y Yanahuaya.

\section{Estado de conservación}

En las localidades evaluadas de la cuenca media del río Tambopata se registraron 16 especies listadas en alguna categoría de amenaza en la legislación peruana (Ministerio de Agricultura 2004), en la lista roja de la Unión Internacional para la Conservación de la Naturaleza y los Recursos Naturales (IUCN 2008), o en los apéndices de la Convención sobre el Comercio Internacional de Especies Amenazadas de flora y fauna (CITES 2010). Estas especies son un oso hormiguero, siete primates, dos roedores, tres carnívoros, un tapir y dos cetartiodáctilos (Tabla 6). De ellas los primates Ateles chamek y Lagothrix cana y el oso de anteojos Tremarctos ornatus son las especies más amenazadas; incluso esta última es considerada en la actualidad como una especie rara por los pobladores locales. El tapir Tapirus terrestris y el oso hormiguero gigante Myrmecophaga tridactyla se encuentran en estado vulnerable; la primera es considerada una especie rara y la segunda una especie común por lo pobladores de la zona. El tigrillo Leopardus pardalis, el puma Puma concolor y el oso de anteojos Tremarctos ornatus son especies considerada como especies en peligro de extinción por el CITES. Mientras que, todos los primates, el oso hormiguero Myrmecophaga tridactyla, el tapir Tapirus terrestres, el sajino Pecari tajacu y la huangana Tayassu pecari registrados en el presente estudio son especies que no están necesariamente amenazadas de extinción pero que podrían llegar a estarlo a menos que se controle estrictamente su comercio. El majaz Cuniculus paca es considerado una especies frecuente y es cazada por los pobladores locales, por lo que podría verse amenazada siendo considerada por el CITES como una especie que cuenta con el apoyo internacional para evitar la explotación insostenible o ilegal de la misma.

Tabla 5. Índices de diversidad, número de especies e individuos capturados para las localidades evaluadas en la cuenca media del río Tambopata. Unidades de vegetación: $\mathrm{CF}=$ cafetal, $\mathrm{PC}=$ pacal, $\mathrm{BPB}=$ bosque pre-montano bajo, $\mathrm{E}=$ ecotono bosque-río, $\mathrm{BPM}=$ bosque pre-montano, $\mathrm{CR}=$ cultivos de cítricos, $\mathrm{DC}=$ diversos cultivos y BM = bosque montano. Índices de diversidad: $\mathrm{H}^{\prime}=$ índice de diversidad de Shannon-Wiener, 1-D = índice de diversidad de Simpson, H' max = índice de diversidad máxima y J' = índice de equidad.

\begin{tabular}{|c|c|c|c|c|c|}
\hline Localidad & San Fermín & Curva Alegre & Challohuma & Yanahuaya & Yanacoha \\
\hline Unidad de vegetación & $\mathrm{CF}, \mathrm{PC}, \mathrm{BPB}$ & $\mathrm{BPB}, \mathrm{E}$ & BPM, CR & $\mathrm{PC}, \mathrm{DC}$ & $\mathrm{BM}$ \\
\hline $\mathrm{N}^{\circ}$ de especies & 27 & 25 & 17 & 13 & 14 \\
\hline $\mathrm{N}^{\circ}$ de individuos & 97 & 189 & 49 & 47 & 79 \\
\hline $\mathrm{H}^{\prime}$ & 4,20 & 3,22 & 3,52 & 2,17 & 3,36 \\
\hline $\mathrm{H}^{\prime} \max$ & 4,75 & 4,64 & 4,09 & 3,70 & 3,81 \\
\hline$J^{\prime}$ & 0.88 & 0.69 & 0.86 & 0.59 & 0.88 \\
\hline 1-D & 0,93 & 0,78 & 0,88 & 0,58 & 0,88 \\
\hline
\end{tabular}


Tabla 6. Especies de mamíferos con su estado de conservación en la cuenca media del río Tambopata. EN: en peligro, VU: Vulnerable; I = Apéndice I, II = Apéndice II. Fuente: Decreto Supremo No. 034-2004AG (Ministerio de Agricultura 2004). Lista roja de la Unión Internacional para la Conservación de la Naturaleza y los Recursos Naturales (IUCN 2008). Apéndices de la Convención sobre el Comercio Internacional de Especies Amenazadas de Flora y Fauna (CITES 2010).

\begin{tabular}{|c|c|c|c|c|c|}
\hline 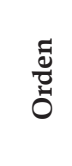 & 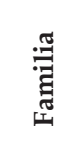 & Especie & $\begin{array}{l}\infty \\
\text { ڤ్̀ } \\
Z \\
己 \\
己\end{array}$ & $\begin{array}{l}\text { तू } \\
\text { ' } \\
\text { مी }\end{array}$ & 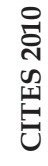 \\
\hline \multicolumn{6}{|c|}{ Cingulata } \\
\hline \multicolumn{6}{|c|}{ Myrmecophagidae } \\
\hline & & Myrmecophaga tridactyla & & VU & II \\
\hline \multicolumn{6}{|c|}{ Primates } \\
\hline \multicolumn{6}{|c|}{ Cebidae } \\
\hline & & Saguinus fuscicollis & & & II \\
\hline & & Aotus azarae & & & II \\
\hline & & Cebus apella & & & II \\
\hline & & Saimiri boliviensis & & & II \\
\hline \multicolumn{6}{|c|}{ Pitheciidae } \\
\hline & & Callicebus aureipalatii & & & II \\
\hline \multicolumn{6}{|c|}{ Atelidae } \\
\hline & & Alouatta sara & & & II \\
\hline & & Ateles chamek & EN & $\mathrm{VU}$ & II \\
\hline & & Lagothrix cana & EN & VU & II \\
\hline \multicolumn{6}{|c|}{ Rodentia } \\
\hline \multicolumn{6}{|c|}{ Cuniculidae } \\
\hline & & Cuniculus paca & & & III \\
\hline \multicolumn{6}{|c|}{ Carnivora } \\
\hline \multicolumn{6}{|c|}{ Felidae } \\
\hline & & Leopardus pardalis & & & I \\
\hline & & Puma concolor & & & I \\
\hline \multicolumn{6}{|c|}{ Ursidae } \\
\hline & & Tremarctos ornatus & VU & EN & I \\
\hline \multicolumn{6}{|c|}{ Perissodactyla } \\
\hline \multicolumn{6}{|c|}{ Tapiridae } \\
\hline & & Tapirus terrestris & VU & VU & II \\
\hline \multicolumn{6}{|c|}{ Cetartiodactyla } \\
\hline \multicolumn{6}{|c|}{ Tayassuidae } \\
\hline & & Pecari tajacu & & & II \\
\hline & & Tayassu pecari & & & II \\
\hline
\end{tabular}

\section{Conservación}

La cuenca del río Tambopata atraviesa el Parque Nacional Bahuaja Sonene y la Reserva Nacional del Tambopata, y albergan una alta diversidad de especies y hábitats que van desde los 400 $\mathrm{m}$ hasta cerca de los $2000 \mathrm{~m}$ de elevación (Ministerio de Agricultura 2003). Lamentablemente la zona media de la cuenca del río Tambopata se ve afectada por un manejo no planificado de las actividades económicas de la región, tales como la deforestación, la minería informal y la producción de monocultivos e.g. de café que traen como consecuencia la pérdida de la diversidad y la degradación de los hábitats. Se ha observado la construcción de carretas que proyectan llegar hasta el borde del Parque Nacional Bahuaja Sonene lo que influiría en un incremento de colonos en la ZA del PNBS con la consecuente alteración del hábitat. También hay indicios y avistamientos de cultivo ilegal de coca. Todos estos hechos son amenazas para la conservación y seguridad de los bosques pre-montanos y yungas de la cuenca media del río Tambopata, los cuales albergan especies importantes para la conservación como el oso de anteojos Tremarctos ornatus; y roedores raros como Oxymycterus juliacae y Lenoxus apicalis. Dado lo mencionado, las zonas exploradas ameritan tener algún grado de protección, por lo que se sugiere la extensión de la zona sur de la ZA del PNBS hasta los bosques montanos de Yanacocha, ya que en la actualidad la ZA protege una muy pequeña porción de los bosque tropicales de altura (Ministerio de Agricultura 2003), los cuales albergan un alto número de especies endémicas y son tan o más diversos que los bosques de la selva baja (Pacheco 2002, Pacheco et al. 2009).

Por otro lado la tala y la quema de bosques por parte de agricultores para establecer los cultivos de café, cítricos y plátanos son problemas adicionales que producen un incremento de la fragmentación en la cuenca media del río Tambopata. Se recomienda establecer una zonificación ecológica y un mayor control del uso de tierras. También se recomienda el uso de agroecosistemas, tales como los cafetales con sombra que pueden ser una buena opción para la conservación de la región por los ingresos obtenidos y por la riqueza de mamíferos relativamente alta que mantiene (Cruz-Lara et al. 2004), y la ejecución de proyectos de educación ambiental.

\section{Discusión}

La parte sureste del Perú es uno de los lugares menos estudiados. Pocos son los esfuerzos realizados en esta zona en cuanto a la investigación de mamíferos resaltando hasta el momento un estudio sobre gradiente de elevación desarrollado en la Reserva de Biósfera del Manu (ver Patterson et al. 1996), que abarcó los bosques de Cusco y Madre de Dios; sin embargo, los bosques de Puno cuentan sólo con menciones de registros de mamíferos pequeños no voladores en muy pocas localidades del departamento, por ejemplo Hinojoza et al. (1987), Da Silva (1998) y Patton y Smith (2002). Las yungas y bosques pre-montanos de Puno no cuentan con estudios detallados de diversidad, los cuales son ahora más importantes que nunca para sustentar nuevos esfuerzos en la conservación de la zona. El presente trabajo representa por ello un esfuerzo inicial de dicho interés.

La importancia biogeográfica de la cuenca media del río Tambopata se demuestra por los numerosos nuevos registros aquí obtenidos. Los marsupiales Marmosa (Micoureus) demerarae, Marmosops bishopi, M. impavidus, el roedor Neacomys musseri y los murciélagos Eumops auripendulus, Platyrrhinus masu, P. nigellus, P. albericoi y Cormura brevirostris son los primeros registros para el departamento de Puno.

Como era de esperarse, San Fermín y Curva Alegre (40 y 38 especies respectivamente), ambos con hábitats de bosques pre-montanos bajos fueron los que presentaron mayor riqueza mientras que Yanahuaya presentó la menor riqueza $(n=33)$. Esto nos indica que la riqueza disminuye con el incremento de la altitud (Tabla 5). Mientras que la diversidad se presenta casi uniforme para todas las localidades (Tabla 5). Por lo tanto en general la localidad más rica y diversa es San Fermín; mientras que Yanahuaya es la localidad con menor riqueza de especies y con los valores más bajos en los índices de H’ y J'. Cabe mencionar que los bajos valores registrados en Yanahuaya pudieron estar influenciados por el bajo número de especies de murciélagos registrados en comparación con el resto de localidades, probablemente influenciado por los días de luna llena durante el muestreo, periodo no recomendado para la captura de quirópteros (Flores 2008). Los datos de riqueza sugieren que los 
bosques montanos albergan un menor número de especies que los bosques pre-montanos bajos; sin embargo los datos de diversidad muestran que todas las localidades evaluadas presentan una diversidad equivalente, al parecer no correlacionada por la altitud. Los altos valores del índice de equidad en San Fermín, Challohuma y Yanacocha, indican que estos lugares están cerca de alcanzar su máxima diversidad, deduciéndose que son los lugares mejores muestreados en la cuenca media del río Tambopata.

La falta de correlación entre la diversidad de mamíferos y la elevación, puede deberse también al corto rango de elevación entre las localidades. Sin embargo, nuestros registros sugieren que el bosque montano presenta especies propias y ausentes en los bosques pre-montanos bajos (e.g., Tremarctos ornatus, Monodelphis peruviana, Akodon baliolus, Lenoxus apicales, Oxymycterus juliacae, Histiotus velatus). Sin duda, las especies pre-montanas van disminuyendo también conforme aumenta la elevación, pero hacen falta más puntos de evaluación a lo largo de la gradiente para documentar este patrón.

Por otro lado, la abundancia relativa (AR) de los mamíferos no voladores y murciélagos si está correlacionada con la elevación, positiva para el primer grupo y negativa para el segundo. Aparte de la elevación, otros factores como la cantidad de recursos disponibles y/o la competencia con otras especies podrían ser los responsables de la abundancia observada. En San Fermín y Yanahuaya se resalta la abundancia de las especies de la subfamilia Carollinae, ya que son considerados como buenos indicadores de hábitats perturbados (Wilson et al. 1996).

Tampoco es sorpresa encontrar que en la cuenca media del río Tambopata los roedores y murciélagos $(67,5 \%)$ son los grupos taxonómicos más diversos, en concordancia con lo expuesto por Pacheco (2002). Sin embargo, el que la familia Phyllostomidae sea la más numerosa, es debido a un efecto de muestreo basado más en el uso de redes. Se sugiere, en el futuro, el empleo de diversos métodos para incorporar especies de otras familias (Voss \& Emmons 1996).

En forma preliminar se concluye que la cuenca del río Tambopata alberga al menos 141 especies de mamíferos, que resulta de incluir las 76 especies aquí registradas y las especies listadas por Ascorra y Orihuela (in litt.) y Foster et al. (1994). Se observa que esta diversidad es mayor a las 130 especies presentes en un rango de 800-3500 m en la Reserva de Biósfera del Manu (ver Solari et al. 2006). Nuestros registros de la cuenca media del río Tambopata representan un poco más de la mitad de las especies presente en toda la cuenca del río Tambopata, mostrando que las Yungas y bosques pre-montanos de la vertiente suroriental son tan o casi tan diversos como las selvas bajas (Ascorra y Orihuela in litt., Pacheco 2002, Pacheco et al. 2009). Esto también demuestra que la zona evaluada se encuentra relativamente bien muestreada.

Este trabajo sugiere la extensión de la zona sur de la ZA del PNBS hasta los bosques montanos de Yanacocha, ya que la ZA de este parque nacional protege una muy pequeña porción de estos bosque tropicales de altura (Ministerio de Agricultura 2003) de esta forma se protegería la diversidad expuesta en este documento. En San Fermín es preocupante el uso de las áreas deforestadas dedicadas al cultivo ilegal de la coca Erythroxylum coca. Young (1996) reportó que la deforestación atribuible a la coca puede llegar tanto como a un millón de hectáreas de bosques pre-montanos en el Perú; pero aunque no existen datos actuales, es evidente que esta cantidad está en aumento. También se ve un gran interés por parte de la población de colonizar la ZA del PNBS hecho corroborado por el avistamiento de carreteras en construcción con miras de adentrarse más a la ZA del parque. Este escenario, aunado al imperceptible esfuerzo de conservación en la zona, amenaza el alto nivel de diversidad y probable endemismo de la cuenca media del río Tambopata.

En conclusión, la alta diversidad de mamíferos de la cuenca media del río Tambopata, la presencia de especies únicas mencionadas anteriormente y la importancia biogeográfica de la zona incentivan a incrementar los estudios en la zona y demandan una mayor atención en la conservación de sus bosques, especialmente en el escenario actual de evidente deforestación e inminente aumento de colonos y rivereńos en la ZA del PNBS.

\section{Agradecimientos}

Al Ministerio de Agricultura por facilitarnos los permisos de colecta correspondientes. A Miryam Quevedo, Jesús Lezcano, Mónica Aguirre, Jhon Morales y Edson Aguilar quienes apoyaron en el trabajo de campo. Esta investigación fue financiada gracias a fondos obtenidos a través del Consejo Superior de Investigación de la Universidad Nacional Mayor de San Marcos y una "Beca María Koepcke" coordinado por Conservación Internacional-CI Perú y la Asociación Peruana para la Conservación de la Naturaleza- APECO.

\section{Literatura citada}

Allen, J.A. 1900. On mammals collected in southeastern Peru, by Mr. H.H. Keays, with descriptions of new species. Bulletin of the American Museum of Natural History, 13: $219-227$.

CITES 2010. (en línea). Apéndices I, II y III. <www.cites.org/esp/ app/appendices.shtml $>$. Acceso 05/11/10.

Cruz-Lara L., C. Lorenzo, L. Soto, E. Naranjo \& N. Ramírez. 2004. Diversidad de mamíferos en cafetales y selva mediana de las cañadas de la selva Lacandona, Chiapas, México. Acta Zoológica Mexicana (n.s) 20(1): 63-81.

da Silva M. N. F. 1998. Four new species of spiny rats of the genus Proechimys (Rodentia: Echimyidae) from the western Amazon of Brazil. Proceedings of the Biological Society of Washington 111: 436-471.

Emmons L.H. \& F. Feer. 1997. Neotropical rainforest mammals, a field guide. 2da edn., The University of Chicago Press, Chicago. Pp. 307.

Flores M. 2008. Estructura de las comunidades de murciélagos en un gradiente ambiental en la reserva de la biosfera y tierra comunitaria de origen Pilón Lajas, Bolivia. Mastozoología Neotropical 15(2): 309-322.

Foster R.B., J.L. Carr \& A.B. Forsyth. 1994. The Tambopata-Candamo Reserved Zone of Southeastern Peru: a biological assessment. Rapid Assessment Program Working Papers $\mathrm{N}^{\circ}$ 6. Conservation International. Pp. 140- 149.

Gardner A.L. (editor). 2008 (2007). Mammals of South America, Volume 1. Marsupials, xenarthrans, shrews, and bats. The University of Chicago Press, Chicago. Pp. 669.

Hinojosa P., F. S. Anderson \& J.L. Patton. 1987. Two new npecies of Oxymycterus (Rodentia) from Peru and Bolivia. American Museum Novitates 2898: 1-17.

IUCN 2008. (en línea). IUCN Red list of threatened species. Version 2010.1. <www.iucnredlist.org > . Acceso 05/11/2010.

Krebs C. J. 1989. Ecological methodology. Harper Collins. New York. Pp 654.

Márquez G. \& V. Pacheco. 2010. Nuevas evidencias de la presencia 
del Oso Andino (Tremarctos ornatus) en las Yungas de Puno, el registro más austral de Perú. Revista Peruana de Biología 17(3): 377 - 380.

Ministerio de Agricultura. 2003. Plan Maestro del Parque Nacional Bahuaja Sonene 2003-2008. Lima- Perú. Pp. 24-42.

Ministerio de Agricultura. 2004. Decreto Supremo No. 034- 2004AG. El Peruano. Pp. 276853-276855.

Nishihara H., M. Hasegawa \& N. Okada. 2006. Pegasoferae, an unexpected mammalian clade revealed by tracking ancient retroposon insertions. Proceedings of the National Academy of Science. 103 (26): 9929-9934.

Nishihara H., S. Maruyamab \& N. Okadaa. 2009. Retroposon analysis and recent geological data suggest near-simultaneous divergence of the three superorders of mammals. Proceedings of the National Academy of Science. 106 (13): 5235-5240.

Osgood, W. H. 1915. New mammals from Brazil and Peru. Field Museum of Natural History. Publ. Zool. Ser. 10: 187-198.

Pacheco V. 2002. Mamíferos del Perú. In: G. Ceballos y J. Simonetti, eds. Diversidad y conservación de los mamíferos neotropicales. Conabio-UNAM. México, D.F. Pp. 503-550.

Pacheco V., S. Solari \& P. M. Velazco. 2004. A new species of Carollia (Chiroptera: Phyllostomidae) from the Andes of Peru and Bolivia. Occasional Papers, Museum of Texas Tech University 236: 1-15.

Pacheco V., E. Salas, L. Cairampoma, et al. 2007a. Diversidad y conservación de los mamíferos en la cuenca del río Apurímac, Perú. Revista peruana de Biología 14(2): 169- 180.

Pacheco V., H.L. Quintana, P.A. Hernandez, et al. 2007b. Mamíferos. In: B.E. Young (editor). Distribución de las especies endémicas en la vertiente oriental de los Andes en Perú y Bolivia. NatureServe, Arlington, Virginia, EE UU. Pp. 40-45.

Pacheco V., R. Cadenillas, E. Salas, et al. 2009. Diversidad y endemismo de los mamíferos del Perú. Revista Peruana de Biología 16(1): 005-032.

Patterson B., V. Pacheco \& S. Solari. 1996. Distribution of bats along an elevational gradient in the Andes of south-eastern Peru. Journal of Zoology 240: 637-658.

Patton J. L. \& M. F. Smith. 1992. Evolution and systematics of the akodontine rodents (Muridae:Sigmodontinae) of Peru, with emphasis on the genus Akodon. Memorias del Museo de Historia Natural, Universidad Nacional Mayor de San Marcos, 21:83-104.
Patton J.L., P. Myers \& M. F. Smith. 1990. Vicariant versus gradient models of diversification: the small mammal fauna of eastern Andean slopes of Peru. Pp. 355-371, in Vertebrates in the Tropics (G. Peters y R. Hutterer, eds.). Zoologisches Forschunginstitut und Museum Alexander Koenig, Bonn, 424 pp.

Smith M.F. y J.L. Patton. 2007. Molecular phylogenetics and diversification of South American grass mice, genus Akodon. Pp. 827-858 in Kelt, D. A., E. P. Lessa, J. Salazar-Bravo, and J. L. Patton (eds.). The Quintessential Naturalist: Honoring the Life and Legacy of Oliver P. Pearson. University of California Publications in Zoology 134:1-981

Solari S. 2007. New species of Monodelphis (Didelphimorphia: Didelphidae) from Peru, with notes on M. adusta (Thomas, 1897). Journal of Mammalogy 88(2): 319-329.

Solari S., V. Pacheco, L. Luna, et al. 2006. Mammals of the Manu Biosphere Reserve. In: B.D. Patterson, D.F. Stotz y S. Solari, Eds. Mammals and birds of the Manu Biosphere Reserve, Peru. Fieldiana Zoology (New Series) 110: 13-22.

Springer M.S., W.J. Murphy, E. Eizirik \& S. J. O’Brien. 2003. Placental mammal diversification and the Cretaceous-Tertiary boundary. Proceedings of the National Academy of Since (PNAS). 100 (3): Pp. 1056-1061.

Van Dijk M., E. Paradis, F. Catzeflis \& W. De Jong. 1999. The virtues of gaps: Xenarthran (edentate) monophyly supported by a unique deletion in a a-crystallin. Systematic biology 48(1): 94-106.

Voss R.S. \& L.H. Emmons. 1996. Mammalian Diversity in Neotropical Lowland rainforest: A preliminary assessment. Bulletin of the American Museum of Natural History 230:18-22.

Wilson D.E., \& D.M. Reeder, eds. 2005. Mammal Species of the World: A Taxonomic and Geographic Reference. 3rd edn. Johns Hopkins University Press, Baltimore. 2142 pp.

Wilson D., C. Ascorra \& S. Solari. 1996. Bats as indicators of habitat disturbance. En Wilson \& A. Sandoval (edit.). MANU: La Biodiversidad del Sureste del Perú. Washington, D.C.: Smithsonian Institution Press. Pp. 613-625.

Young K. R. 1996. Threats to biological diversity caused by coca/ cocaine deforestation in Peru. Environmental Conservation 23: 7-15.

Apéndice 1. Lista de especies de mamíferos registrados en toda el área del Tambopata. Localidades: $Y A C O=$ Yanacocha, YANA $=$ Yanahuaya, CHAL = Challohuma, CURV = Curva alegre, FERS = San Fermín. Reportes previos: 1= Foster et al. (1994), 2= Ascorra y Orihuela, in litt. Tipo de registro: $A$ = avistamiento, $C=$ captura, $E=$ entrevista, $H=$ huella, $V=$ vocalización, $H e=$ heces, $M=$ marcas. $E^{1}$ determinación tentativa.

\begin{tabular}{|c|c|c|c|c|c|c|c|}
\hline \multirow[b]{3}{*}{ Nombre científico } & \multirow[b]{3}{*}{ Nombre común } & \multicolumn{6}{|c|}{ Cuenca del río Tambopata } \\
\hline & & \multicolumn{5}{|c|}{ Cuenca media } & $\begin{array}{c}\text { Cuenca } \\
\text { baja }\end{array}$ \\
\hline & & 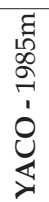 & 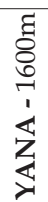 & 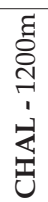 & 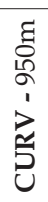 & 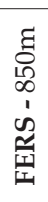 & $\begin{array}{c}\text { Reportes } \\
\text { previos } \\
<700 \mathrm{~m}\end{array}$ \\
\hline
\end{tabular}

\begin{tabular}{lllc}
\hline \multicolumn{2}{l}{ Didelphimorphia } & & \\
\hline \multicolumn{2}{c}{ Didelphidae } & Zarigüeyita lanuda & 1,2 \\
\hline 1 & Caluromys lanatus (Olfers, 1818) & Zarigüeyita de estola negra & 2 \\
\hline 2 & Caluromysiops irrupta Sanborn, 1951 & Zarigüeya orejinegra & 1,2 \\
\hline 3 & Didelphis marsupialis Linnaeus, 1758 & Comadrejita marsupial radiante & 2 \\
\hline 4 & Marmosa lepida (Thomas, 1888) &
\end{tabular}




\section{Nombre científico}

\begin{tabular}{cl} 
& Nombre científico \\
& \\
\hline 5 & Marmosa rubra Tate, 1931 \\
\hline 6 & Marmosa (Micoureus) demerarae Thomas, 1905 \\
\hline 7 & Marmosa (Micoureus) regina Thomas, 1898 \\
\hline 8 & Marmosops bishopi (Pine, 1981) \\
\hline 9 & Marmosops impavidus (Tschudi, 1845) \\
\hline 10 & Marmosops noctivagus (Tschudi, 1844) \\
\hline 11 & Metachirus nudicaudatus (É. Geoffroy, 1803) \\
\hline 12 & Monodelphis peruviana (Osgood, 1913) \\
\hline 13 & Philander opossum (Linnaeus, 1758) \\
\hline
\end{tabular}

Nombre común

Comadrejita marsupial rojiza

Comadrejita marsupial lanuda

Comadrejita marsupial reina

Comadrejita marsupial de Bishop

Comadrejita marsupial pálida

Comadrejita marsupial noctámbula

Rata marsupial de cuatro ojos

Colicorto marsupial peruano

Zarigüeyita gris de cuatro ojos

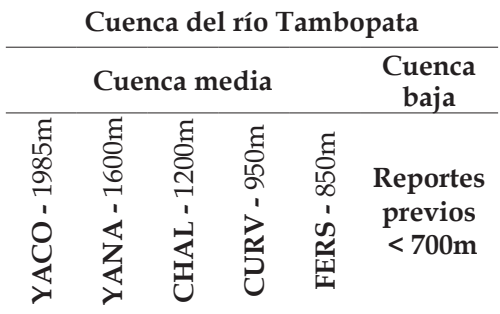

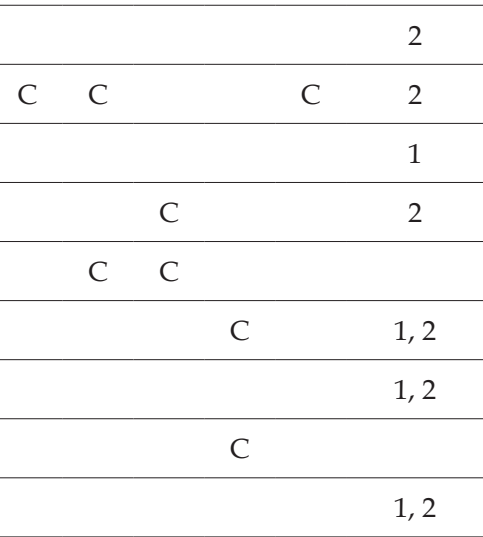

Xenarthra

\begin{tabular}{lc}
\hline \multicolumn{2}{c}{ Dasypodidae } \\
\hline 14 & Dasypus kappleri Krauss, 1862 \\
\hline 15 & Dasypus novemcinctus Linnaeus, 1758 \\
\hline 16 & Cabassous unicinctus (Linnaeus, 1758) \\
\hline 17 & Priodontes maximus (Kerr, 1792)
\end{tabular}

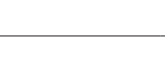

Armadillo de Kappler

1,2

Armadillo de nueve bandas,

carachupa

$\mathrm{E}^{1}$

Armadillo de cola desnuda

Armadillo gigante

1

1

1

1,2

Bradypodidae

18 Bradypus variegatus Schinz, 1825

Perezoso de tres dedos

Megalonychidae

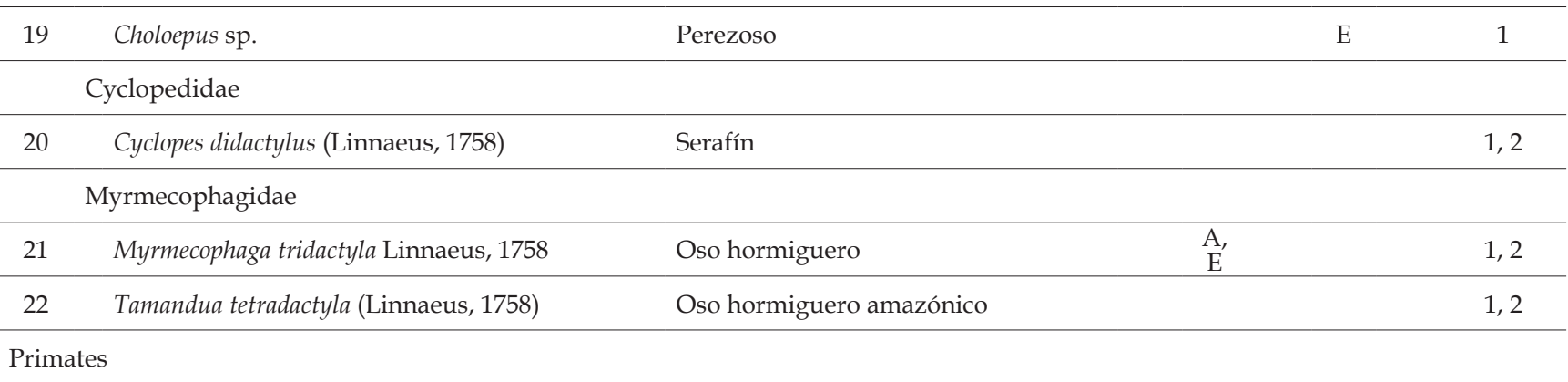

Cebidae

\begin{tabular}{|c|c|c|c|c|c|c|c|}
\hline 23 & Saguinus fuscicollis (Spix, 1823) & Pichico común & & A & & A & 1,2 \\
\hline 24 & Aotus azarae (Humboldt, 1811) & Mono nocturno de Azara & E & $\mathrm{E}$ & & & 1,2 \\
\hline 25 & Cebus albifrons (Humboldt, 1812) & Machín frontiblanco, machín blanco & & & & & 1,2 \\
\hline 26 & Cebus apella (Linnaeus, 1758) & Machín negro & & A & $\mathrm{E}$ & A & 1,2 \\
\hline 27 & Saimiri boliviensis (I.Geoffroy y Blainville, 1834) & Monofraile boliviano, frailecillo & & & $\mathrm{E}$ & A & 1,2 \\
\hline \multicolumn{8}{|c|}{ Pitheciidae } \\
\hline 28 & $\begin{array}{l}\text { Callicebus aureipalatii Wallace, Gómez, A.Felton } \\
\& \text { A. M. Felton, } 2006\end{array}$ & Tocón del Madidi & & A & & & \\
\hline 29 & Callicebus brunneus (Wagner, 1842) & Tocón moreno & & & & & 1,2 \\
\hline \multicolumn{8}{|c|}{ Atelidae } \\
\hline 30 & Alouatta sara Elliot, 1910 & Mono aullador rojo boliviano & & & $\begin{array}{l}\mathrm{A}, \\
\mathrm{E}, \\
\mathrm{V}\end{array}$ & $\begin{array}{c}\text { A, } \\
\text { V }\end{array}$ & 1,2 \\
\hline
\end{tabular}

(Continúa...) 


\begin{tabular}{|c|c|c|c|c|c|c|c|c|}
\hline 31 & Ateles chamek (Humboldt, 1812) & Mono araña negro, maquisapa & & & & & $\mathrm{A}$ & 1,2 \\
\hline 32 & Lagothrix cana (É. Geoffroy, 1812) & Mono lanudo gris & & & & E & & \\
\hline \multicolumn{9}{|c|}{ Rodentia } \\
\hline \multicolumn{9}{|c|}{ Sciuridae } \\
\hline 33 & Microsciurus flaviventer (Gray, 1867) & Ardillita de vientre amarillo & & & & & $\mathrm{A}$ & \\
\hline 34 & Sciurus ignitus (Gray, 1867) & Ardilla ígnia & & & & & A & 1,2 \\
\hline 35 & Sciurus spadiceus Olfers, 1818 & Ardilla baya & & & & & A & 1,2 \\
\hline \multicolumn{9}{|c|}{ Cricetidae } \\
\hline 36 & Akodon baliolus Osgood, 1915 & & $\mathrm{C}$ & $\mathrm{C}$ & $\mathrm{C}$ & & & \\
\hline 37 & Euryoryzomys nitidus (Thomas, 1884) & Ratón arrozalero lustroso & & & & $\mathrm{C}$ & $\mathrm{C}$ & \\
\hline 38 & Hylaeamys perenensis (J. A. Allen, 1901) & Ratón arrozalero cabezudo & & $\mathrm{C}$ & & & $\mathrm{C}$ & 1,2 \\
\hline 39 & Lenoxus apicalis (J. A. Allen, 1900) & Rata andina & $\mathrm{C}$ & $\mathrm{C}$ & & & & \\
\hline 40 & $\begin{array}{l}\text { Neacomys musseri Patton, da Silva y Malcolm, } \\
2000\end{array}$ & Ratón espinoso de Musser & & & & & $\mathrm{C}$ & \\
\hline 41 & Neacomys spinosus (Thomas, 1882) & Ratón espinoso común & $\mathrm{C}$ & & $\mathrm{C}$ & & & 1,2 \\
\hline 42 & Nectomys apicalis Peters, 1861 & Nectomys de la Amazonía occidental & & & & & & 1 \\
\hline 43 & Nephelomys keaysi (J. A. Allen, 1900) & Ratón arrozalero de las yungas & & $\mathrm{C}$ & & & & \\
\hline 44 & Oecomys bicolor (Tomes, 1860) & Ratón arrozalero pardo & & & $\mathrm{C}$ & & $\mathrm{C}$ & 1,2 \\
\hline 45 & Oligoryzomys destructor (Tschudi, 1844) & Ratón arrozalero destructor & $\mathrm{C}$ & $\mathrm{C}$ & $\mathrm{C}$ & & & \\
\hline 46 & Oligoryzomys microtis (J. A. Allen, 1916) & Ratón arrozalero de oreja pequeña & & & & & & 1,2 \\
\hline 47 & Oxymycterus juliacae J. A. Allen, 1900 & Ratón hocicudo de Puno & & & $\mathrm{C}$ & & & \\
\hline 48 & $\begin{array}{l}\text { Rhipidomys gardneri Patton, da Silva y Malcom, } \\
2000\end{array}$ & Rata trepadora de Gardner & & & & & & 2 \\
\hline \multicolumn{9}{|c|}{ Erethizontidae } \\
\hline 49 & Coendou sp. & Puerco espín & $\mathrm{E}$ & $\mathrm{E}$ & & E & & 1,2 \\
\hline \multicolumn{9}{|c|}{ Caviidae } \\
\hline 50 & Galea musteloides Meyen, 1832 & Sasha-cuy & & $\mathrm{E}$ & & & & \\
\hline 51 & Hydrochoerus hydrochaeris (Linnaeus, 1766) & Ronsoco & & & & & & 1,2 \\
\hline \multicolumn{9}{|c|}{ Dasyproctidae } \\
\hline 52 & Dasyprocta variegata Tschudi, 1845 & Añuje, agutí & & & A & E & & 1,2 \\
\hline 53 & Myoprocta pratti Pocock, 1913 & Punchana, añuje menor & & & & & & 1,2 \\
\hline \multicolumn{9}{|c|}{ Cuniculidae } \\
\hline 54 & Cuniculus paca (Linnaeus, 1766) & Majaz, picuro & $\mathrm{E}$ & & $\begin{array}{l}\mathrm{E}, \\
\mathrm{H}\end{array}$ & & & 1,2 \\
\hline \multicolumn{9}{|c|}{ Echimyidae } \\
\hline 55 & Dactylomys boliviensis Anthony, 1920 & Cono-cono boliviano (Anthony) & & $\begin{array}{l}\text { A, } \\
\text { E, } \\
\text { V }\end{array}$ & & $\begin{array}{l}\text { A, } \\
\text { E, } \\
\text { V }\end{array}$ & $\mathrm{V}$ & 1,2 \\
\hline 56 & Dactylomys dactylinus (Desmarest, 1817) & Cono-cono amazónico & & & & & & 1 \\
\hline
\end{tabular}


Nombre científico
Cuenca del río Tambopata

Cuenca media

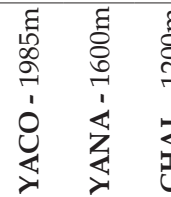

\begin{tabular}{lll}
\hline 57 & Isothrix bistriata Wagner, 1845 & Rata de doble estría, coconocono \\
\hline 58 & Mesomys hispidus (Desmarest, 1817) & Rata espinosa áspera de río Madeira \\
\hline 59 & Proechimys brevicauda (Gunther, 1877) & Rata espinosa colicorta \\
\hline 60 & Proechimys simonsi Thomas, 1900 & Rata espinosa de Simons \\
\hline
\end{tabular}

Lagomorpha

\begin{tabular}{l} 
Leporidae \\
\hline $61 \quad$ Sylvilagus brasiliensis (Linnaeus, 1758)
\end{tabular}

Chiroptera

Emballonuridae

\begin{tabular}{lll}
62 & Rhynchonycteris naso (Wied-Neuwied, 1820) & Murcielaguito narigudo \\
\hline 63 & Saccopteryx bilineata (Temminck, 1838) & Murcielaguito negro de listas
\end{tabular}

Phyllostomidae

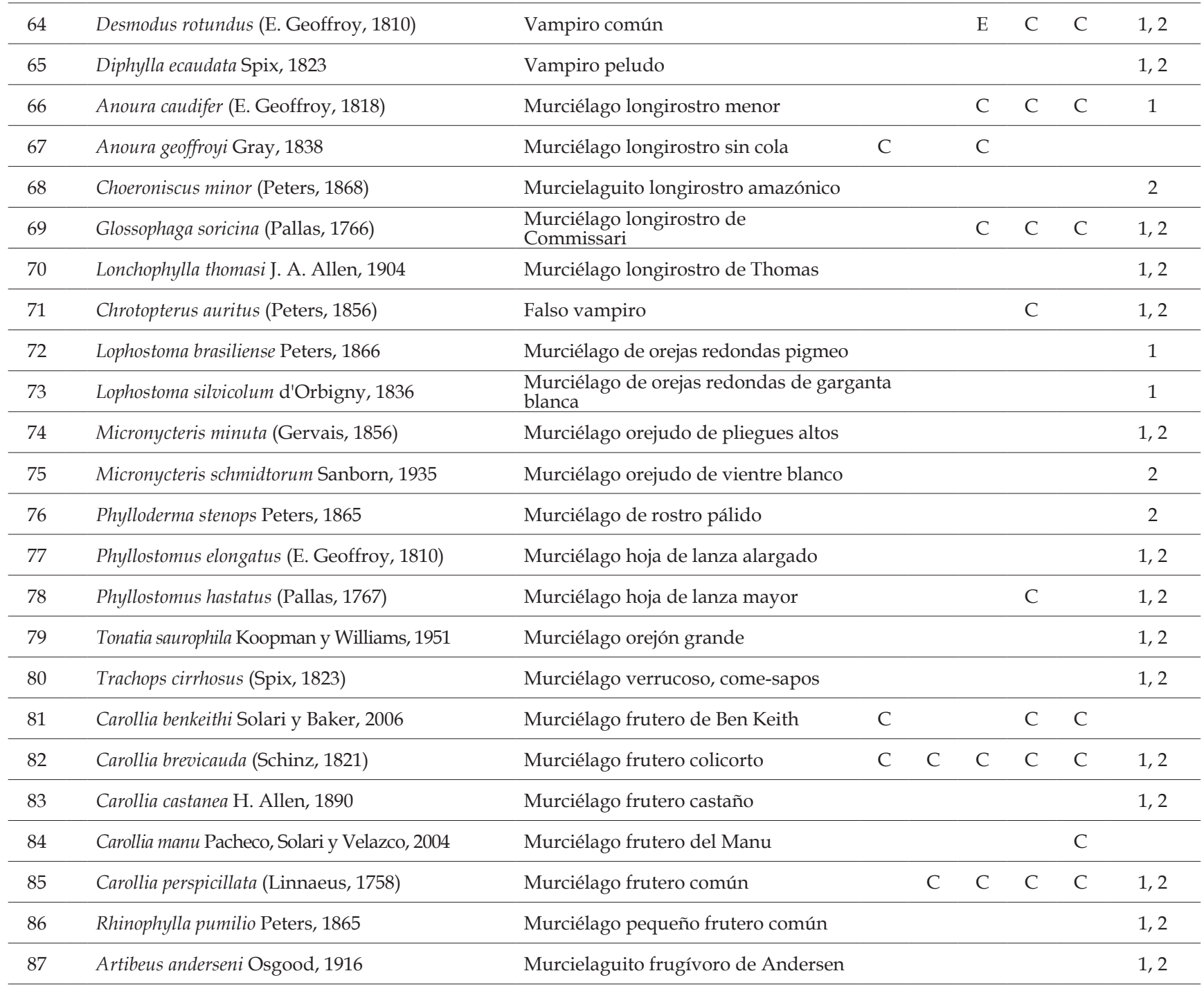




\begin{tabular}{|c|c|c|c|c|c|c|c|c|}
\hline & \multirow[b]{3}{*}{ Nombre científico } & \multirow[b]{3}{*}{ Nombre común } & \multicolumn{6}{|c|}{ Cuenca del río Tambopata } \\
\hline & & & \multicolumn{5}{|c|}{ Cuenca media } & \multirow{2}{*}{$\begin{array}{c}\begin{array}{c}\text { Cuenca } \\
\text { baja }\end{array} \\
\begin{array}{c}\text { Reportes } \\
\text { previos } \\
<700 \mathrm{~m}\end{array}\end{array}$} \\
\hline & & & 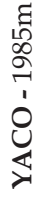 & 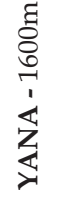 & 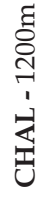 & 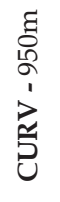 & 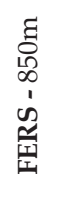 & \\
\hline 88 & Artibeus glaucus Thomas, 1893 & Murciélago frutero plateado & $\mathrm{C}$ & $\mathrm{C}$ & $\mathrm{C}$ & $\mathrm{C}$ & $\mathrm{C}$ & \\
\hline 89 & Artibeus lituratus (Olfers, 1818) & Murcielaguito frugívoro mayor & & & & & $\mathrm{C}$ & 1 \\
\hline 90 & Artibeus obscurus (Schinz, 1821) & Murcielaguito frugívoro negro & & & & & $\mathrm{C}$ & 1,2 \\
\hline 91 & Artibeus planirostris (Spix, 1823) & Murciélago frutero de rostro plano & $\mathrm{C}$ & $\mathrm{C}$ & $\mathrm{C}$ & $\mathrm{C}$ & $\mathrm{C}$ & 1,2 \\
\hline 92 & Chiroderma salvini Dobson, 1878 & Murciélago de listas claras & & & C & & & 1 \\
\hline 93 & Chiroderma trinitatum Goodwin, 1958 & Murciélago menor de listas & & & & $\mathrm{C}$ & $\mathrm{C}$ & \\
\hline 94 & Chiroderma villosum Peters, 1860 & Murciélago de lineas tenues & & & & & & 1,2 \\
\hline 95 & Mesophylla macconnelli Thomas, 1901 & Murcielaguito cremoso & & & & & $\mathrm{C}$ & 1,2 \\
\hline 96 & Platyrrhinus albericoi Velazco, 2005 & $\begin{array}{l}\text { Murciélago de nariz ancha de } \\
\text { Alberico }\end{array}$ & & & & $\mathrm{C}$ & C & \\
\hline 97 & Platyrrhinus brachycephalus (Rouk y Carter, 1972) & $\begin{array}{l}\text { Murciélago de nariz ancha de cabeza } \\
\text { pequeña }\end{array}$ & & & & $\mathrm{C}$ & $\mathrm{C}$ & \\
\hline 98 & Platyrrhinus incarum (Thomas, 1912) & Murciélago de nariz ancha inca & & & & $\mathrm{C}$ & $\mathrm{C}$ & 1,2 \\
\hline 99 & Platyrrhinus infuscus (Peters, 1880) & $\begin{array}{l}\text { Muciélago de nariz ancha de listas } \\
\text { tenues }\end{array}$ & & & & & $\mathrm{C}$ & 2 \\
\hline 100 & Platyrrhinus masu Velazco, 2005 & Murciélago de nariz ancha quechua & $\mathrm{C}$ & $\mathrm{C}$ & & $\mathrm{C}$ & $\mathrm{C}$ & \\
\hline 101 & Platyrrhinus nigellus Gardner y Carter, 1972 & Murciélago de nariz ancha negrito & & & & $\mathrm{C}$ & & \\
\hline 102 & Sturnira erythromos (Tschudi, 1844) & Murciélago frugívoro oscuro & $\mathrm{C}$ & & & C & & \\
\hline 103 & Sturnira lilium (E. Geoffroy, 1810) & Murciélago de charreteras amarillas & $\mathrm{C}$ & $\mathrm{C}$ & $\mathrm{C}$ & $\mathrm{C}$ & $\mathrm{C}$ & 1,2 \\
\hline 104 & Sturnira magna de la Torre, 1966 & $\begin{array}{l}\text { Murciélago de hombros amarillos } \\
\text { grande }\end{array}$ & & & & $\mathrm{C}$ & & 1,2 \\
\hline 105 & Sturnira oporaphilum (Tschudi, 1844) & $\begin{array}{l}\text { Murciélago de hombros amarillos de } \\
\text { oriente }\end{array}$ & $\mathrm{C}$ & & $\mathrm{C}$ & $\mathrm{C}$ & $\mathrm{C}$ & \\
\hline 106 & Sturnira tildae de la Torre, 1959 & Murciélago de charreteras rojizas & & & & & & 1,2 \\
\hline 107 & Uroderma bilobatum Peters, 1866 & Murciélago constructor de toldos & & & $\mathrm{C}$ & $\mathrm{C}$ & $\mathrm{C}$ & 1 \\
\hline 108 & Uroderma magnirostrum Davis, 1968 & $\begin{array}{l}\text { Murciélago amarillento constructor } \\
\text { de toldos }\end{array}$ & & & & & & 1,2 \\
\hline 109 & Vampyrodes caraccioli (Thomas, 1889) & Muciélago de listas pronunciadas & & & & & & 1,2 \\
\hline \multicolumn{9}{|c|}{ Noctilionidae } \\
\hline 110 & Noctilio albiventris Desmarest, 1818 & Murciélago pescador menor & & & & & & 1 \\
\hline 111 & Noctilio leporinus (Linnaeus, 1758) & Murciélago pescador mayor & & & & & & 1 \\
\hline \multicolumn{9}{|c|}{ Thyropteridae } \\
\hline 112 & Thyroptera tricolor Spix, 1823 & $\begin{array}{l}\text { Murciélago de ventosas de vientre } \\
\text { blanco }\end{array}$ & & & & & & 1 \\
\hline \multicolumn{9}{|c|}{ Molossidae } \\
\hline 113 & Eumops auripendulus $($ Shaw, 1800) & Murciélago de cola libre común & & & $\mathrm{C}$ & & & \\
\hline 114 & Tadarida brasiliensis (I. Geoffroy, 1824) & Murciélago mastín & $\mathrm{C}$ & & $\mathrm{C}$ & & & 1 \\
\hline \multicolumn{9}{|c|}{ Vespertilionidae } \\
\hline 115 & Histiotus velatus (I.Geoffroy, 1824) & Murciélago orejón del Trópico & & & C & & & \\
\hline 116 & Lasiurus ega (Gervais, 1856) & Murciélago amarillento & & & & & & 1,2 \\
\hline 117 & Myotis albescens (E. Geoffroy, 1806) & Murcielaguito plateado & & & C & & & 1 \\
\hline 118 & Myotis nigricans (Schinz, 1821) & Murciélago negruzco común & & & & & C & 1 \\
\hline 119 & Myotis riparius Handley, 1960 & Murcielaguito acanelado & & & & & & 1,2 \\
\hline
\end{tabular}


Nombre científico

Nombre común

Murciélago vespertino aterciopelado

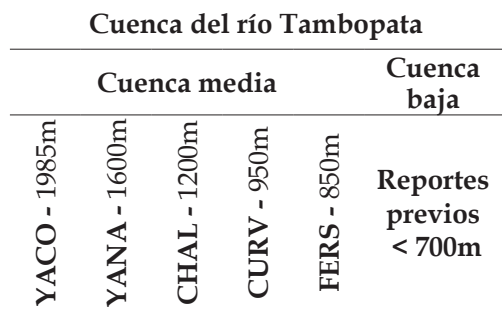

\begin{tabular}{|c|c|c|c|c|c|c|c|c|}
\hline 120 & Myotis simus Thomas, 1901 & Murciélago vespertino aterciopelado & & & & & & 2 \\
\hline \multicolumn{9}{|c|}{ Carnivora } \\
\hline \multicolumn{9}{|c|}{ Felidae } \\
\hline 121 & Leopardus pardalis (Linnaeus, 1758) & Ocelote, tigrillo & E & & & & & 1,2 \\
\hline 122 & Leopardus wiedii (Schinz, 1821) & Margay & & & & & & 1,2 \\
\hline 123 & Puma concolor (Linnaeus, 1771) & Puma & $\mathrm{E}$ & $\mathrm{E}$ & $\mathrm{E}$ & $\mathrm{E}$ & & 1,2 \\
\hline 124 & Puma yagouaroundi (É. Geoffroy Saint-Hilaire,1803) & Yahuarundi & & & & & & 1 \\
\hline 125 & Panthera onca (Linnaeus, 1758) & Jaguar, otorongo & & & & & & 1,2 \\
\hline \multicolumn{9}{|c|}{ Canidae } \\
\hline 126 & Atelocynus microtis (Sclater, 1883) & Zorro negro orejicorto & & & & & & 1,2 \\
\hline 127 & Speothos venaticus (Lund, 1842) & Perro de monte, perro de bosque & & & & & & 1 \\
\hline \multicolumn{9}{|c|}{ Ursidae } \\
\hline 128 & Tremarctos ornatus (F. G. Cuvier, 1825) & Oso de anteojos & $\begin{array}{c}\mathrm{He}, \\
\mathrm{E}\end{array}$ & & M & & & \\
\hline \multicolumn{9}{|c|}{ Mustelidae } \\
\hline 129 & Lontra longicaudis (Olfers, 1818) & Lobo pequeño de río, nutria & & & & & & 1,2 \\
\hline 130 & Pteronura brasiliensis (Gmelin, 1788) & Lobo grande de río, nutria grande & & & & & & 1,2 \\
\hline 131 & Eira barbara (Linnaeus, 1758) & Tejón, manco & & & & & & 1,2 \\
\hline 132 & Galictis vittata (Schreber, 1776) & Hurón grande & & & & & & 1 \\
\hline \multicolumn{9}{|c|}{ Procyonidae } \\
\hline 133 & Bassaricyon alleni Thomas, 1880 & Olingo, chosna & & & & & & 1,2 \\
\hline 134 & Nasua nasua (Linnaeus, 1766) & Coatí de cola anillada, mishasho & & & & & & 1,2 \\
\hline 135 & Potos flavus (Schreber, 1774) & Chosna, cuchumli & & & & & & 1,2 \\
\hline 136 & Procyon cancrivorus (G. [Baron] Cuvier, 1798) & Osito cangrejero & & & & & $\mathrm{H}$ & 1 \\
\hline \multicolumn{9}{|c|}{ Perissodactyla } \\
\hline \multicolumn{9}{|c|}{ Tapiridae } \\
\hline 137 & Tapirus terrestris (Linnaeus, 1758) & Tapir del llano amazónico, sachavaca & & & & & $\mathrm{H}$ & 1,2 \\
\hline \multicolumn{9}{|c|}{ Cetartiodactyla } \\
\hline \multicolumn{9}{|c|}{ Tayassuidae } \\
\hline 138 & Pecari tajacu (Linnaeus, 1758) & Sajino & & & & E & & 1,2 \\
\hline 139 & Tayassu pecari (Link, 1795) & Pecarí, huangana & $\mathrm{E}$ & $\mathrm{E}$ & $\mathrm{E}$ & $\mathrm{E}$ & & 1,2 \\
\hline \multicolumn{9}{|c|}{ Cervidae } \\
\hline 140 & Mazama americana (Erxleben, 1777) & Venado colorado & $\mathrm{E}$ & $\mathrm{E}$ & & $\mathrm{E}$ & $\mathrm{H}$ & 1,2 \\
\hline 141 & Mazama nemorivaga (F. Cuvier, 1817) & Venado gris & & & & & & 1,2 \\
\hline Total & denes & & 6 & 6 & 6 & 8 & 7 & \\
\hline Total & milias & & 11 & 10 & 12 & 14 & 11 & \\
\hline Total & pecies & & 23 & 20 & 32 & 38 & 39 & \\
\hline
\end{tabular}

